ablen Membran hervorgebracht würde, aber auch kleiner als der, welcher dem nicht diffundierenden Anteil für sich zukäme*. Die Ansicht von Kratky und $\mathrm{Mark}^{52}$, daß niedrigmolekulare Anteile sich bei osmotischen Messungen gleichmäßig auf beide Seiten der Membran verteilen und den osmotischen Druck nicht beeinflussen, trifft somit nur für den Grenzfall unendlicher Verdünnung zu. Gl. (28) läßt

s6 Fortschr. Chem. Phys. Techn. makromol. Stoffe 2, 49 [1942].

* Anm. b. d. Korrektur: Hierdurch dürfte sich die Bemerkung von G. V. Schulz Z. physik. Chem. Abt. B 52, schließlich erkennen, daß auch die Konzentrationsfunktion des osmotischen Druckes durch den Verdrängungseffekt in einer ziemlich unübersichtlichen Weise beeinflußt wird. Schon aus diesem Grunde erscheint die von G. V. Schulz $z^{56}$ ausgesprochene Ansicht berechtigt, daß osmotische Messungen zu verwerfen sind, bei welchen Diffusion durch die Membran stattgefunden hat.

1 [1942]) erklären daß der Fehler im osmotischen Druck um eine Größenordnung höher ist als der in der Meßperiode hinausdiffundierende Anteil.

\title{
Über einige Eigenschaften gelöster Fadenmoleküle, III. Mitteilung: Die Solvatation der Fadenmoleküle (Zur Theorie der Lösungen hochpolymerer Substanzen IV)
}

\author{
Von Arnold Münster \\ Aus dem Chemischen Institut der Universität Heidelberg \\ (Z. Naturforschg. 2 a, 284-296 [1947]; eingegangen am 17. Februar 1947)
}

\begin{abstract}
Die experimentellen Abweichungen von der Theorie der athermischen Lösung werden besprochen und es wird gezeigt, daß sie ohne Ausnahme durch die thermodynamische Theorie der Solvatation erklärt werden. Es wird thermodynamisch untersucht, unter welchen Bedingungen die Theorie der idealen Lösung in Verbindung mit der Solvatation nicht zur Deutung der experimentellen Befunde ausreicht.

Die statistische Theorie der Solvatation wird bis zur ersten Näherung entwickelt; es werden Formeln für die Verdünnungswärme, die Verdünnungsentropie und den osmotischen Druck aufgestellt. An Hand der experimentellen Daten für die Verdünnungswärme wird der Gültigkeitsbereich der Näherung untersucht und die zwischenmolekulare Wechselwirkungsenergie berechnet. Der Einfluß der Solvatation auf die Verdünnungsentropie wird im Zusammenhang mit dem Vorzeichen der Verdünnungswärme und der Beweglichkeit der Fadenmoleküle diskutiert. Die auf diesem Wege berechnete athermische Verdünnungsentropie ist in Ubereinstimmung mit der Theorie der athermischen Lösung.

Die gewonnenen Daten ermöglichen bei Kautschuklösungen die vollständige Vorausberechnung des osmotischen Druckes in Übereinstimmung mit der Erfahrung.
\end{abstract}

\section{Einleitung}

$\mathrm{D}_{1}$ e thermodynamischen Eigenschaften gelöster Fadenmoleküle (FM) lassen sich in zahlreichen Fällen durch die in früheren Arbeiten ${ }^{1,2,3}$ entwickelte Theorie der athermischen Lösung erklären. Es gibt indessen verschiedene experimentelle Ergebnisse, zu deren Deutung dieselbe nicht ausreicht. Die wichtigsten, auf die z.Tl. schon früher ${ }^{2,3}$ hingewiesen wurde, sind die folgenden:

1 A. M ünster, Kolloid-Z. 105, 1 [1943].

${ }^{2}$ A. M ü nster, Z. Naturforschg. 1, 311 [1946].

3 A. Münster, Z. Naturforschg. 2a, 272 [1947].
1. Die Verdünnungsentropie wurde bei den Systemen Nitrocellulose-Aceton ${ }^{4,5}$ und PolystyrolToluol $^{4,5}$ innerhalb der Fehlergrenzen gleich dem für die ideale Lösung geltenden Wert gefunden. Das gleiche gilt bei niedrigen Konzentrationen für das System Acetylcellulose-Tetrachloräthan ${ }^{6}$, während bei höheren Konzentra-

4-G. V. Schulz, Z. physik. Chem. (A) 180, 1 [1937].

${ }^{5}$ G. V. Schulz, Z. physik. Chem. (B) 40,319 [1938].

${ }^{6}$ O. H a g g e r u. A. J. A. van der Wy k, Helv. chim. Acta 23, 484 [1940]. 
tionen die Verdünnungsentropie hier sogar negativ wird.

2. Der osmotische Druck zeigt in diesen Fällen die für hochpolymere FM charakteristischen Abweichungen vom van 't $\mathrm{H}$ of f schen Gesetz. Bei Systemen mit anomaler Verdünnungsentropie kann man aus den Meßdaten den Störungsparameter $\alpha_{n}$ ermitteln ${ }^{2}$ und daraus den osmotischen Druck $P$ berechnen ${ }^{3}$. Tab. 1 zeigt, daß die so berechneten Werte $P_{\text {ber }}$ nur bei dem athermischen System Guttapercha-Toluol mit den experimentellen Daten übereinstimmen; dagegen sind bei positiver Verdünnungswärme (Kautschuk-Toluol) die gemessenen osmotischen Drucke kleiner, bei negativer (NitrocelluloseCyclohexanon) größer als die berechneten.

\begin{tabular}{|c|c|c|c|}
\hline System & $\begin{array}{c}c_{g} \\
\mathrm{~g} / \mathrm{l}\end{array}$ & $\begin{array}{c}P_{\text {ber }} 10^{3} \\
\text { Atm. }\end{array}$ & $\begin{array}{c}P_{\text {exp }} 10^{3} \\
\text { Atm. }\end{array}$ \\
\hline Guttapercha-Toluol $^{7}$ & 25,07 & 41,9 & 42,2 \\
& 36,08 & 73,5 & 69,2 \\
Kautschuk-Toluol $^{8}$ & 30,81 & 42,9 & 29,8 \\
& 41,78 & 77,5 & 50,9 \\
Nitrocellulose- $_{\text {Cyclohexanon }}{ }^{9}$ & 16,27 & 9,0 & 16,0 \\
& 32,31 & 28,2 & 50,2 \\
& 61,14 & 81,4 & 150 \\
\hline
\end{tabular}

Tab. 1. Berechnung des osmotischen Druckes $P$ aus der Verdünnungsentropie $\left(c_{g}=\right.$ Gewichtskonzentration $)$.

3. Bei den Systemen Nitrocellulose-Aceton ${ }^{\mathbf{1 0}}$ und Cellulosetriacetat-Chloroform ${ }^{11}$ kann der $\mathrm{Ab}$ solutwert des Störungsparameters $\alpha_{n}$ nicht mehr aus der Theorie der athermischen Lösung abgeleitet werden ${ }^{3}$.

4. Bei Nitrocellulose wurde eine starke Abhängigkeit des osmotischen Druckes vom Lösungsmittel (LM) gefunden ${ }^{12}$.

5. Die experimentell gefundene Abhängigkeit des osmotischen Druckes von der Temperatur kann nicht durch die Theorie der athermischen Lösung erklärt werden ${ }^{3}$.

6. Bei den Systemen Methylcellulose-Wasser ${ }^{13}$ und Triacetylcellulose-Dioxan ${ }^{14}$ steigen $\operatorname{die} P / c_{g}-c_{g}$ Kurven nicht monoton an, sondern sie durchlaufen Extremwerte.

7 E. W o lff, Helv. chim. Acta 23, 439 [1940].

$8 \mathrm{~K}$. H. Meyer, E. Wolff u. Ch. G. B o is s o n n as, Helv. chim. Acta 23, 430 [1940].

9 Ch. G. B o is sonn as u. K. H. M e y e r, Helv. chim. Acta 20, 783 [1937].

10 G. V. Schulz, Z. physik. Chem. (A) 176, 317 [1936].
Im folgenden soll untersucht werden, inwieweit die Solvatation, d. h. die energetische Wechselwirkung der FM mit dem LM, die Eigenschaften der athermischen Lösung modifiziert und ob sie die obigen Ergebnisse zu erklären vermag.

\section{Thermodynamische Theorie der Solvatation}

Um die Solvatation thermodynamisch $\mathrm{zu}$ formulieren, geht man zweckmäßig aus von der Gi b b s-H e l m h o l t z schen Gleichung ${ }^{15}$, die für die freie Energie der Verdünnung $\Delta \mu_{2}$ lautet

$$
\Delta \mu_{2}-T \frac{\partial \Delta \mu_{2}}{\partial T}=\Delta H_{2}
$$

( $\Delta_{H_{2}}=$ Verdünnungswärme). Die Integration ergibt

( $R=$ Gaskonstante) .

$$
\Delta \mu_{2}-R T \eta=-T \int_{\infty}^{T} \frac{\Delta H_{2}}{T^{2}} d T
$$

Die Integrationskonstante $R \eta$ ist die durch die Temperatur dividierte freie Energie der Verdünnung bei unendlich hoher Temperatur. Da hier alle Konfigurationen die gleiche Energie besitzen, ist dieser Ausdruck identisch mit dem aus der Theorie der athermischen Lösung berechneten $\left(\Delta \mu_{2} / T\right)_{\text {ath }}$. Wird für das Integral die Abkürzung $-R \varphi$ eingeführt, so erhält man

$$
\Delta \mu_{2}=R T\left[\eta\left(n_{1}, n_{2}\right)+\varphi\left(n_{1}, n_{2}, T\right)\right]
$$

$\left(n_{1}, n_{2}=\right.$ Molzahlen der FM und des LM). Die Funktion $\varphi$ stellt das durch die Solvatation bedingte Zusatzglied zur Theorie der athermischen Lösung dar. Aus Gl. (3) folgt sofort die Verdünnungsentropie

$$
\Delta s_{2}=-R\left[\eta+\varphi+T \frac{\partial \varphi}{\partial T}\right]
$$

oder mit Gl. (2)

$$
\Delta s_{2}=-R \eta+T \int_{\infty}^{T} \frac{\Delta H_{2}}{T^{2}} d T+\frac{\Delta H_{2}}{T} .
$$

Für die Verdünnungswärme hat man

$$
\Delta H_{2}=-R T^{2} \frac{\partial \varphi}{\partial T} .
$$

11 H. S t a u d i n ger u. G. D a u m i 11 e r, Liebigs Ann. Chem. 529, 219 [1937].

12 A. D o b r y, Kolloid-Z. 81, 190 [1937].

13 G. V. S c h u l z, Z. physik. Chem. (A) 177, 453 [1936].

${ }_{14}$ F. K unz e, Z. physik. Chem. 188, 90 [1941].

15 G. N. L ewis u. M. R and a ll, Thermodynamik, deutsch v. O. R e d l i ch, Wien [1927]. 
Da für den osmotischen Druck $P$ die Beziehung

$$
\Delta \mu_{2}=-\int_{p}^{p+P} V_{2} d p=-\bar{V}_{2} P
$$

gilt ${ }^{15}$, wo $V_{2}$ das partiale Molvolumen des LM und $p$ der Druck ist, folgt aus den Gln. (2) und

$$
P=-\frac{R T}{\bar{V}_{2}}(\eta+\varphi)
$$

oder

$$
P=-\frac{R T}{\overline{V_{2}}} \eta+\frac{T}{V_{2}} \int_{\infty}^{T} \frac{\Delta H_{2}}{T^{2}} d T .
$$

Schließlich ergibt sich für den logarithmischen Temperaturkoeffizienten des osmotischen Druckes

$$
\frac{\partial \ln P}{\partial \ln T}=1-\frac{T}{\bar{V}_{2}} \frac{\partial \bar{V}_{2}}{\partial T}+T \frac{\partial \varphi}{\partial T}(\eta+\varphi)^{-1}
$$

oder

$$
\frac{\partial \ln P}{\partial \ln T}=1-\frac{T}{\bar{V}_{2}} \frac{\partial \bar{V}_{2}}{\partial T}+\frac{\Delta H_{2}}{P \bar{V}_{2}} .
$$

Aus den Gln. (4a) und (7a) folgt, daß die Abweichung von der idealen Lösung im Falle $\Delta_{\mathbf{2}}>0$ für die Verdünnungsentropie größer ist als für den osmotischen Druck, während im Falle $\Delta_{H_{2}}<0$ das Gegenteil zutrifft (Tab.1). Im letzteren Falle ist es somit auch möglich, daß die Verdünnungsentropie gleich der idealen oder sogar kleiner gefunden wird, obwohl der Betrag des osmotischen Druckes über dem van 't H of f schen Gesetz liegt $(\mathrm{I}, 1)$. Dieses Ergebnis ist daher kein Argument gegen die Theorie der athermischen Lösung. Der strenge Nachweis, daß $\eta$ den aus dieser Theorie folgenden Wert besitzt, kann allerdings nur erbracht werden, indem entweder das Integral auf Grund experimenteller Daten ausgewertet oder aber die $\varphi$-Funktion theoretisch ermittelt und über $\Delta_{H_{2}}$ an das Experiment angeschlossen wird. Der erste Weg ist heute noch nicht gangbar, da das erforderliche Versuchsmaterial nicht vorliegt. Die statistische Berechnung der $\varphi$-Funktion wird im folgenden Abschnitt versucht werden.

Nach Gl. (7a) ist es ohne weiteres verständlich, daß der osmotische Druck noch über den aus der Theorie der athermischen Lösung berechneten Wert hinaus wachsen oder Extremwerte durchlaufen kann, sowie daß er vom LM abhängt. Aus Gl. (8a) folgt (wenn das kleine Volumenglied vernachlässigt wird), daß für $\Delta H_{2} \gtrless 0$

$$
\frac{\Delta P}{P} \mid \frac{\Delta T}{T} \gtrless 1
$$

ist. Dies stimmt, wie schon früher ${ }^{3}$ gezeigt, mit der Erfahrung überein.

Die quantitative Auswertung der Gl. (8a) ist ohne weiteres möglich, hat aber nur Sinn, wenn die Verdünnungswärmen unabhängig von dem osmotischen Druck (kalorimetrisch) bestimmt sind. Bei dem vorliegenden Versuchsmaterial trifft dies nicht zu.

Damit ist nachgewiesen, daß sich in der Tat alle in der Einleitung angeführten Ergebnisse durch die Solvatation deuten lassen. Man kann nun noch fragen, ob nicht die Solvatation allein zur Erklärung der thermodynamischen Eigenschaften gelöster FM ausreicht. Diese Frage läßt sich, wie oben erwähnt, thermodynamisch zwar im Prinzip beantworten, doch reicht dazu das vorliegende experimentelle Material nicht aus. Macht man aber die Voraussetzung

$$
\left|\frac{\Delta H_{2}}{T}\right|=\left|\Delta H_{2} \int_{\infty}^{T} \frac{d T}{T^{2}}\right|>\left|\int_{\infty}^{T} \frac{\Delta H_{2}}{T^{2}} d T\right|,
$$

so kann man zeigen, daß $\eta>\ln \left(1-N_{1}\right)\left(N_{1}=\mathrm{Mo}^{-}\right.$ lenbruch der FM) sein $m u ß$, daß sich also die nach Abspaltung der Solvatation verbleibende Verdünnungsentropie nicht nach der Theorie der idealen Lösung berechnen läßt. Es sind dann folgende Fälle möglich:

1. $\int_{\infty}^{T} \frac{\Delta H_{2}}{T^{2}} d T$ und $\frac{\Delta H_{2}}{T}$ haben entgegengesetztes
Vorżeichen.

a) $\Delta H_{2}>0$.

Wäre $\eta=\ln \left(1-N_{1}\right)$, so müßte nach $\mathrm{Gl}$. (7a) der osmotische Druck kleiner als bei der idealen Lösung und nach Gl. (4a) $-R \ln \left(1-N_{1}\right)+\Delta H_{2} / T>\Delta s_{2}$ sein. Beides trifft nicht $\mathrm{zu}$.

b) $\Delta H_{2}<0$.

Hier müßte $\Delta s<-R \ln \left(1-N_{1}\right)$ sein, was nur bei dem System Triacetylcellulose-Tetrachloräthan in höheren Konzentrationen gefunden wurde.

$$
T
$$

2. $\int^{T} \frac{\Delta H_{2}}{T^{2}} d T$ und $\frac{\Delta H_{2}}{T}$ haben gleiches Vorzeichen.

a) $\Delta H_{2}>0$.

Nach den experimentellen Daten müßte, wenn $\eta=\ln \left(1-N_{1}\right)$ wäre, das Integral ein Mehrfaches von $\Delta_{2} / T$ sein, was nach der Voraussetzung nicht zutrifft. 
b) $\Delta H_{2}<0$.

$\eta=\ln \left(1-N_{1}\right)$ ist nur möglich, wenn osmotischer Druck und Verdünnungsentropie kleiner sind als bei der idealen Lösung, was nicht der Fall ist.

Unter der Voraussetzung (9) gibt es somit keine Annahme über das Integral, die gleichzeitig mit den Versuchsergebnissen und $\eta=\ln \left(1-N_{1}\right)$ $\mathrm{zu}$ vereinbaren ist.

\section{Statistische Theorie der Solvatation*}

\section{a) Der allgemeine Ansatz}

Für die freie Energie (nach $\mathrm{Helmholtz}$ ) einer binären Lösung liefert die statistische Mechanik $^{16,17}$ die Formel

$$
\begin{aligned}
A_{L}^{0}=-k T\left[N_{1}\left(\ln \frac{G_{1}{ }^{\prime}}{N_{1}}+1\right)\right. \\
\left.\quad+N_{2}\left(\ln \frac{G_{2}{ }^{\prime}}{N_{2}}+1\right)+\ln B(T)\right]
\end{aligned}
$$

mit

$$
B(T)=\int \ldots \int \exp (-E / k T) d \tau
$$

( $k=\mathrm{B}$ oltzmann sche Konstante, $N_{1}=\mathrm{Zahl}$ der gelösten Moleküle, $N_{2}=$ Zahl der Moleküle des LM, $G^{\prime}=$ Verteilungsfunktion der kinetischen und inneren Energie der Moleküle, $B(T)=$ Verteilungsfunktion der potentiellen Energie des Gesamtsystems, $E=$ potentielle Energie des Gesamtsystems, $d \tau=$ Volumenelement des Phasenraumes).

Die Berechnung des Integrals Gl. (11) beruht, wie schon früher ${ }^{2}$ erörtert, zunächst auf der durch das Experiment gestützten Annahme, daß $E$ scharfe Minima besitzt für gewisse Konfigurationen, bei welchen sich alle Moleküle innerhalb eines gewissen Volumens $V$ befinden. Für alle übrigen Konfigurationen wird $E$ praktisch unendlich; ihr Beitrag zum Integral kann vernachlässigt werden. Jede Konfiguration in den Minima kann man nach Guggen hei m ${ }^{17}$ sowie Fow ler und Rushbrooke ${ }^{18}$ auffassen als Überlagerung einer gitterartigen „Standard-Konfiguration" mit den zwischenmolekularen Schwingungen usw. In der Theorie der athermischen Lö-

* Anm. b. d. Korrektur: Das Problem ist kürzlich auch von E. A. Gug gen heim (Proc. Roy. Soc. London (A) 183, 213 [1944]), W. J. C. O r r (Trans. Faraday Soc. 40, 320 [1944]), T. A 1 f r e y u. P. M. D o t y (J. Chem. Physics 13, 77 [1945]) behandelt worden. Nur die letztere Arbeit war mir nach Abschluß dieser Untersuchung im Original zugänglich. sung ${ }^{1,2}$ wurde angenommen, daß alle StandardKonfigurationen die gleiche Energie $E_{\min }$ besitzen und $\mathrm{da}$ die Verteilungsfunktionen der inner- und zwischenmolekularen Schwingungen usw. für alle Standard-Konfigurationen gleich sind. Ferner wurde noch angenommen, daß auch außerhalb der Standard-Konfigurationen die Energie nicht wesentlich über $E_{\text {min }}$ ansteigt, daß also das zwischenmolekulare Potential die Gestalt eines Topfes mit flachem Boden und steilen Wänden habe. Diese Annahme ist nicht notwendig und ohne Einfluß auf das Resultat, aber zur Formulierung bequem und soll daher beibehalten werden.

Man kann nun die Solvatation statistisch dadurch definieren, daß infolge der energetischen Wechselwirkung zwischen den FM und den Molekülen des LM nicht mehr alle Standard-Konfigurationen die gleiche Energie und die gleichen Verteilungsfunktionen für zwischenmolekulare Schwingungen usw. besitzen. Es existieren daher verschiedene Minima von $E$, auch $E_{\text {min }}$ ist als Funktion der Phasenkoordinaten anzusetzen. Um unter dieser allgemeineren Voraussetzung das Integral der Gl. (11) zu berechnen, mögen folgende Annahmen eingeführt werden:

1. In der Lösung treten keine weitreichenden Kräfte auf. Eine energetische Wechselwirkung findet nur zwischen unmittelbar benachbarten Gitterpunkten statt.

2. Die Lösung sei so verdünnt, daß bei der Mehrzahl der Standard-Konfigurationen benachbarte Gitterpunkte nicht von Bausteinen verschiedener FM besetzt werden. Alle diese StandardKonfigurationen besitzen die gleiche Energie $E_{0}$.

Auf Grund dieser Annahme kann man zunächst setzen

und weiterhin

$$
E_{\min }=E_{0}+E_{s}
$$

$B(T)=\exp \left(-E_{0} / k T\right) \int \cdots \int \exp \left(-E_{s} / k T\right) d \tau$.

In dem größeren Teil des Phasenraumes (welcher überhaupt zum Integral beiträgt) ist $E_{\text {min }}$ $=E_{0}=$ const. Das Gebiet, in welchem $E_{s} \neq 0$ ist, werde mit $\tau_{s}$ bezeichnet. Es umfaßt alle StandardKonfigurationen, bei welchen benachbarte Gitter-

16 R. H. F ow le r, Statistische Mechanik, deutsch v. O. H a l p e r n u. H. S m e r e k e r, Leipzig [1931]. 17 E. A. Gug ge nh e i m, Proc. Roy. Soc. [London] (A) 135, 181 [1932].

18 Fow le r u. Rushbrooke, Trans. Faradav Soc. 33, 1272 [1937]. 
punkte von Bausteinen verschiedener FM besetzt sind. Trennt man das Integral über dieses Gebiet ab, so erhält man

$$
B(T)=\exp \left(-E_{0} / k T\right) \int \ldots \int d \tau+\exp \left(-E_{0} / k T\right) \int_{\left(\tau_{s}\right)} \ldots \int\left[\exp \left(-E_{s} / k T\right)-1\right] d \tau .
$$

Die Bedeutung dieser Umformung liegt darin, daß die Energie $E_{0}$ als Bezugsniveau eingeführt und die Verteilungsfunktion $B(T)$ in das athermische ${ }^{2}$ und das Solvatationsglied zerlegt wird.

Die nächste Annahme lautet:

3. Die Lösung sei so verdünnt, daß alle StandardKonfigurationen vernachlässigt werden können, bei welchen Bausteine von mehr als zwei verschiedenen FM benachbarte Gitterpunkte besetzen.

Durch 1. und 3. ist das Phasengebiet $\tau_{s}$ eindeutig definiert. Es umfaßt alle Standard-Konfigurationen, bei welchen „Paare“ von FM auftreten, d.h. je zwei FM mit wenigstens je einem Baustein benachbarte Gitterpunkte besetzen. Die Zahl dieser Paare im Phasengebiet $\tau_{s}$ ist wenigstens 1 und höchstens $N_{1} / 2$.

4. Bei der Zerlegung des Solvatationsintegrals in die Anteile der Standard-Konfigurationen und. der zwischenmolekularen Schwingungen kann der letztere in erster Näherung vernachlässigt werden.

Diese Annahme läßt sich präziser formulieren, wenn man als Modell für die zwischenmolekularen Schwingungen einen linearen harmonischen Oszillator annimmt. Für diesen lautet die $\mathrm{Ha}$ mil t o n sche Funktion

$$
H_{0}=v_{0} J,
$$

wo $v_{0}$ die Frequenz und $J$ die Wirkungsvariable ist. Den Ersatz eines LM-Moleküls durch den Baustein eines FM kann man als Störung auffassen und für die gestörte $\mathrm{H}$ a milt o n - Funktion ansetzen ${ }^{16}$

$$
H_{1}=v_{0} J+\overline{\delta \Omega},
$$

wo $\overline{\delta \Omega}$ die Änderung der potentiellen Energie im Zeitmittel ist. Da für den linearen harmonischen Oszillator gilt

$$
\Omega=\frac{1}{2} k x^{2}, \quad \delta \Omega=\frac{x^{2}}{2} \delta k,
$$

hat man

$$
\overline{\delta \Omega}=\delta \Omega_{0}+\frac{\delta k}{\tau} \int_{0}^{\tau} \frac{x^{2}}{2} d \tau
$$

wo $\tau$ die Periode ist und $\delta \Omega_{0}$ die Änderung der potentiellen Energie in der Ruhelage. Das ergibt

$$
\overline{\delta \Omega}=\delta \Omega_{0}+\frac{1}{4} \delta k x_{\max }^{2} .
$$

Da für $x_{\max }$ die kinetische Energie verschwindet, muß gelten

$$
H_{0}=v_{0} J=\frac{1}{2} k x_{\text {max }}^{2}
$$

und somit

$$
\overline{\delta \Omega}=\delta \Omega_{0}+\frac{1}{2} J v_{0} \frac{\delta k}{k},
$$

oder wegen $v_{0} \sim \sqrt{k}$ und $\frac{1}{2} \frac{\delta k}{k}=\frac{\delta \nu}{v_{0}}$

$$
\overline{\delta \Omega}=\delta \Omega_{0}+J \delta \nu .
$$

Die 4. Annahme besagt dann $\delta \Omega_{0} \gg J \delta v$.

Damit reduziert sich die Aufgabe auf die Berechnung der Summe

$$
Z=\underset{\tau_{s}}{\Sigma}\left[\exp \left(-E_{s} / k T\right)-1\right],
$$

die über alle Standard-Konfigurationen mit $E_{s} \neq 0$ $\mathrm{zu}$ erstrecken ist.

\section{b) Die Zahl der Konfigurationen mit $r$ Paaren}

Die Forderung, daß in der Lösúng $r$ Paare vorhanden sind, wird durch eine große Zahl von Anordnungen der Gesamtlösung, die mit $g_{r}$ bezeichnet werde, erfüllt. Andererseits umfaßt der Begriff des Paares bei FM selbst wieder eine große Zahl von Konfigurationen: Wenn $n$ der Polymerisationsgrad ist, können 1 bis $n$ Bausteine benachbarte Gitterpunkte besetzen, und jede dieser Möglichkeiten kann wieder durch verschiedene Anordnungen realisiert werden. Man kann daher, wenn $E_{r}^{*}$ die Energie des $r$-ten Paares (bezogen auf $E_{0}$ ) ist und $\nu$ die Zahl der Konfigurationen eines Paares, die Gl. (23) schreiben

$Z=\sum_{r=1}^{N_{1} / 2} g_{r} \sum_{v_{1}} \ldots \sum_{\nu_{r}}\left\{\exp \left[-\sum_{r} E_{r}^{*} / k T\right]-1\right\}$.

Die $r$-fache Summierung ist nur über Konfigurationen $\mathrm{zu}$ erstrecken, für welche alle $E_{r} \neq 0$ sind. Es werde zunächst die Größe $g_{r}$ und die Summierung über $r$ betrachtet. $g_{r}$ setzt sich aus zwei Anteilen zusammen: Der Zahl der Möglichkeiten für die Bildung von $r$ Paaren und der 
Zahl der Konfigurationen einer Lösung, welche $r$ Paare enthält (wobei diese jetzt gegeben sind und die Konfigurationen innerhalb der Paare noch nicht gezählt werden). Um diese Abzählung durchzuführen, ist es zweckmäßig, etwa die Lösung mit einem Paar aufzufassen als eine Lösung von $N_{1}-1 \mathrm{FM}$, zu der ein weiteres, das „gepaarte“, hinzugefügt wird.

Bei insgesamt $N_{1}$ FM kann zunächst jedes den Platz des gepaarten einnehmen. Dies ergibt einen Faktor $N_{1}$ in $g_{1}$. Von den übrigen $N_{1}-1$ FM kann wieder jedes das gepaarte bei sich haben, was einen Faktor $N_{1}-1$ bedingt. Schließlich ist noch mit $1 / 2 \mathrm{zu}$ multiplizieren, um die doppelte Zählung auszuschließen. Bei zwei Paaren hat man für das erste wieder $1 / 2 N_{1}\left(N_{1}-1\right)$ Möglichkeiten, für das zweite dann noch $1 / 2\left(N_{1}-2\right)$ $\left(N_{1}-3\right)$. Da die Vertauschung der Bezeichnung des ersten und zweiten Paares keine neue Anordnung ergibt, ist noch durch 2 ! zu dividieren. Für die Herstellung von $r$ Paaren gibt es also

$$
\left(\frac{1}{2}\right)^{r} \frac{N_{1} !}{r !\left(N_{1}-2 r\right) !}
$$

Möglichkeiten. Dieser Faktor ist bereits aus der Theorie der realen Gase ${ }^{16}$ bekannt.

Da $r$ FM durch die Bedingung, daß sie gepaart sein sollen, fixiert sind, bleibt noch die Zahl der Konfigurationen einer Lösung mit $N_{1}-r \mathrm{FM} \mathrm{zu}$ bestimmen. Den Ausdruck dafür liefert die Theorie der athermischen Lösung ${ }^{1,2}$. Damit jedoch nicht mehr als $r$ Paare auftreten, ist hier die Zahl der virtuellen FM so zu bestimmen, daß die einem reellen FM benachbarten Gitterpunkte nicht von Bausteinen eines FM besetzt werden können. Die so berechnete Größe werde mit $\Lambda_{r}$ bezeichnet. Man erhält dann

$g_{r}=\frac{\left(N_{1}-r+\Lambda_{r}\right) ! N_{2} !}{\Lambda_{r} !} \frac{N_{1} !}{r !\left(N_{1}-2 r\right) !}\left(\frac{1}{2}\right)^{r}$.

Der Summenfaktor in Gl. (24) lautet für ein Paar

$$
\sum_{\nu}\left\{\exp \left[-E^{*} / k T\right]-1\right\}
$$

Bei zwei Paaren hat man

$$
\sum_{v_{1}} \sum_{2}\left\{\exp \left[-\left(E_{1}^{*}+E_{2}^{*}\right) / k T\right]-1\right\} .
$$

Dafür kann man schreiben

$$
\sum_{v_{1}} \sum_{v_{2}}\left\{\left[\exp \left(-E_{1}{ }^{*} / k T\right)-1\right]\left[\exp \left(-E_{2}{ }^{*} / k T\right)-1\right]+\left[\exp \left(-E_{1}{ }^{*} / k T\right)-1\right]+\left[\exp \left(-E_{2}{ }^{*} / k T\right)-1\right]\right\} \text {. }
$$

Die beiden letzten Terme entsprechen den Konfigurationen der Gruppe von zwei Paaren, für welche jeweils $E_{1}{ }^{*}$ oder $E_{2}{ }^{*}$ (aber nicht $E_{1}{ }^{*}+E_{2}{ }^{*}$ ) verschwindet. Diese sind bereits in der Summierung für ein Paar gezählt. Sie unterliegen nicht der Doppelsummierung und entfallen nach der bei Gl. (24) gegebenen Vorschrift. Es bleibt daher

$$
\left\{\sum_{\nu}\left[\exp \left(-E^{*} / k T\right)-1\right]\right\}^{2}
$$

Durch analoge Anwendung dieser Betrachtung erhält man schließlich

$$
Z=\sum_{r=1}^{N_{1} / 2} \frac{\left(N_{1}-r+\Lambda_{r}\right) ! N_{2} !}{\Lambda_{r} !} \frac{N_{1} !}{r !\left(N_{1}-2 r\right) !}\left(\frac{1}{2}\right)^{r}\left\{\sum_{\nu}\left[\exp \left(-E^{*} / k T\right)-1\right]\right\}^{r} .
$$

In dem ersten Faktor treten nur große Zahlen auf. Man kann daher die Stirling sche Formel darauf anwenden. Da ferner die Solvatation hier nur als Effekt zweiter Ordnung berechnet wird und auch der Störungseffekt der athermischen Lösung $^{2}$ ein Effekt zweiter Ordnung ist, kann letzterer bei der Berechnung der Solvatation hier vernachlässigt werden. Berücksichtigt man noch, daß nach Voraussetzung 2 und $3 N_{1}-r \ll \Lambda_{r}$, $N_{1} \ll \Lambda\left[\Lambda=\right.$ Zahl der virtuellen FM nach $\left.{ }^{2}\right]$ und $n N_{1} \ll N_{2}$ ist, so kann man $\ln \left(N_{1}-r+\Lambda_{r}\right)$ durch $\ln \left(N_{1}+\Lambda\right), A_{r} \ln \left(N_{1}-r+\Lambda_{r}\right) / \Lambda_{r}-\left(N_{1}-r\right)$ durch $\Lambda \ln \left(N_{1}+\Lambda\right) / \Lambda-N_{1}$ ersetzen und erhält dann

$$
Z=\exp \left\{N_{1} \ln \left(N_{1}+\Lambda\right)+\Lambda \ln \left(N_{1}+\Lambda\right) / \Lambda+N_{2} \ln N_{2}-N_{1}-N_{2}\right\} \sum_{r=1}^{N_{1} / 2} \frac{N_{1} !}{r !\left(N_{1}-2 r\right) ! N_{1}^{r}} x^{r}
$$


mit

$$
\varkappa=\frac{N_{1}}{N_{2}} \frac{1}{2 f(z)} \sum_{v}\left[\exp \left(-E^{*} / k T\right)-1\right],
$$

wo $f(z)$ die Zahl der virtuellen FM ist, die sich von einem Gitterpunkt aus aufbauen lassen. Da der Ausdruck unter dem Summenzeichen in Gl. (27) für $r=0$ den Wert 1 annimmt, kann man, wenn der Anteil von $B(T)$ für die Standard-Konfiguration mit $B^{\prime}(T)$ bezeichnet wird, setzen

$$
\begin{aligned}
B^{\prime}(T)=\exp \left(E_{0} / k T\right) \exp \left\{\mathrm{N}_{1} \ln \left(N_{1}+\Lambda\right)+\Lambda \ln \left(N_{1}+\Lambda\right) / A+N_{2} \ln N_{2}-N_{1}-N_{2}\right\} \\
\sum_{r=0}^{N_{1} / 2} \frac{N_{1} !}{r !\left(N_{1}-2 r\right) ! N_{1}^{r}} \varkappa^{r},
\end{aligned}
$$

wo $x$ nach Gl. (28) und Voraussetzung 2 und 3 klein ist. Wie U r e $11^{19}$ gezeigt hat, kann man eine Funktion

$$
F=\sum_{r=0}^{N / 2} \frac{N !}{r !(N-2 r) ! N^{r}} x^{r}
$$

für kleine $x$ approximieren durch

mit

$$
F=\exp N g
$$

$$
g=x+0\left(x^{2}\right) .
$$

Es wird somit

$$
\begin{aligned}
\ln B^{\prime}(T)= & -\frac{E_{0}}{k T}+N_{1} \ln \left(N_{1}+\Lambda\right) \\
& +\Lambda \ln \frac{N_{1}+\Lambda}{\Lambda}+N_{2} \ln N_{2}-N_{1}-N_{2} \\
& +\frac{N_{1}^{2}}{N_{2}} \frac{1}{2 f(z)} \sum_{v}\left[\exp \left(E^{*} / k T\right)-1\right] .
\end{aligned}
$$

Im folgenden werde die Abkürzung

$$
\Phi=\sum_{v}\left[\exp \left(-E^{*} / k T\right)-1\right]
$$

benutzt.

$\Phi_{\mathrm{starr}}=(z-2)\left\{n^{2}[\exp (-\varepsilon / k T)-1]-2 \exp \left(-n \varepsilon / h^{2}\right.\right.$

\section{c) Die Konfigurationen eines Paares}

Die Konfigurationen eines Paares lassen sich zunächst in Gruppen unterteilen nach der Zahl der benachbarten Bausteine. Alle Konfigurationen einer solchen Gruppe besitzen die gleiche Energie

$$
E^{*}=m_{\varepsilon},
$$

wo $m$ die Zahl der benachbarten Bausteine und $\varepsilon$ die auf das Niveau $E_{0}$ bezogene Wechselwirkungsenergie zweier Bausteine verschiedener FM ist.

Die auf das gleiche Niveau wie $E_{0}$ bezogene Wechselwirkungsenergie zweier Bausteine $\varepsilon_{11}$ hängt mit $\varepsilon$ zusammen durch die Beziehung

$$
\varepsilon=\varepsilon_{11}-2 \varepsilon_{12}+\varepsilon_{22}
$$

$\left(\varepsilon_{12}=\right.$ Wechselwirkungsenergie zwischen Baustein und LM-Molekül, $\varepsilon_{22}=$ Wechselwirkungsenergie zwischen zwei LM-Molekülen).

Die Zahl der Konfigurationen einer Gruppe ist abhängig von der Beweglichkeit der FM. Es werde zunächst der Fall betrachtet, daß dieselben starr sind. Hier ist die Zahl der Konfigurationen einer Gruppe für $m=2$ bis $m=n-14(z-2)$, wo $z$ die Zahl der unmittelbaren Nachbarn eines Gitterpunktes ist. Für $m=n$ hat man $2(z-2)$ Konfigurationen und für $m=1 \quad(z-2) n^{2}$. Es wird somit, wenn man noch berücksichtigt, daß $n \gg 1$ ist,
Bei ideal beweglichen $\mathrm{FM}^{2}$ sind die Konfigurationen jeder Gruppe noch mit einem weiteren Faktor zu multiplizieren.

Es seien etwa $m$ Bausteine benachbart. Die Lage derselben innerhalb des FM sei bei dem einen zunächst beliebig fixiert. Legt man sie bei den anderen an den Anfang des FM, so kommen infolge der Beweglichkeit $(z-1)^{n-m}$ Anordnungen hinzu. Rückt man die gepaarten Bausteine um ein Glied weiter, so

${ }^{19}$ U r s e ll, Proc. Cambridge philos. Soc. 23, 685 [1927]. hat man zusätzlich $(z-1)(z-1)^{n-m-1}$ Anordnungen, rückt man um zwei weiter, $(z-1)^{2}$ $\cdot(z-1)^{n-m-2}$ usw. Die gleiche Überlegung kann man auch für das andere FM anstellen. Der gesamte Faktor der $m$-ten Gruppe lautet somit

$$
(n-m+1)^{2}(z-1)^{2(n-m)} .
$$

Bei dieser Abzählung sind Konfigurationen, bei welchen die $m$ Bausteine nicht zusammenhängen, sowie die Störung der beiden FM vernachlässigt. Man hat also jetzt 


$$
\begin{aligned}
& \Phi_{\text {ideal bewegl }}=(z-2)\left\{2 \sum_{m=1}^{n}(n-m+1)^{2}(z-1)^{2(n-m)}[\exp (-m \varepsilon / k T)-1]\right. \\
& \left.-n^{2}(z-1)^{2(n-1)}[\exp (-\varepsilon / k T)-1]\right\} \text {. }
\end{aligned}
$$

FM, die eine geringere Beweglichkeit besitzen, kann man in starre Segmente von je $x$ Bausteinen zerlegen, die durch Gelenke mit idealer ,Beweglichkeit verbunden sind ${ }^{3}$. Dann gilt, wenn $\varepsilon>0$ ist, näherungsweise

$$
\Phi_{\text {schwach bewegl }}=(z-2) n^{2}(z-1)^{2}\left(\frac{n}{x}\right)-1 \exp (-\varepsilon / k T) .
$$

\section{d) Die thermodynamischen Funktionen}

Aus Gl. (10) und (33) erhält man, wie früher ${ }^{2}$ im einzelnen gezeigt, für das thermodynamische Potential der Lösung

$$
\begin{aligned}
F_{L}=R T\left[n_{1} \ln \frac{n_{1}}{n_{1}+\lambda}+\lambda \ln \frac{\lambda}{n_{1}+\lambda}-n_{1} \ln G_{1}-n_{2} \ln G_{2}+\frac{1}{2} \frac{(z-2)\left(E_{11}-E\right) n n_{1}}{R T}\right. & \\
& \left.+\frac{1}{2} \frac{z E_{22} n_{2}}{R T}-\frac{\Phi}{2 f(z)} \frac{n_{1}{ }^{2}}{n_{2}}+\frac{p}{R} \frac{\mathrm{V}_{0}}{T}\left(1-\frac{1}{2} x p\right)\right]
\end{aligned}
$$

$(\lambda=$ Molzahl der virtuellen FM, $G=$ Verteilungsfunktionen der inner- und zwischenmolekularen Schwingungen etc., $\mathrm{V}_{0}=$ Volumen der Lösung beim Druck $p=0, x=$ Kompressibilität der Lösung, $E_{11}, E_{22}$ und $E=$ Wechselwirkungsenergie pro Mol). Für das chemische Potential der FM folgt daraus (wenn das Glied mit $x$ vernachlässigt und das gewöhnliche Volumen eingesetzt wird)

$$
\mu_{1}=R T\left[\ln \gamma+\frac{\partial \lambda}{\partial n_{1}} \ln (1-\gamma)-\ln G_{1}+\frac{1}{2} \frac{(z-2) n\left(E_{11}-E\right)}{R T}-\frac{\Phi}{f(z)} \frac{n_{1}}{n_{2}}+\frac{p V_{1}}{R T}\right]
$$

und für das chemische Potential des LM

$$
\mu_{2}=R T\left[\frac{\partial \lambda}{\partial n_{2}} \ln (1-\gamma)-\ln G_{2}+\frac{1}{2} \frac{z E_{22}}{R T}+\frac{\Phi}{2 f(z)} \frac{n_{1}{ }^{2}}{n_{2}{ }^{2}}+\frac{p V_{2}}{R T}\right]
$$

$\left(\gamma=\frac{n_{1}}{n_{1}+\lambda}=\right.$ virtueller Molenbruch $^{2} ; \quad V_{1}, V_{2}=$ molare Partialvolumina $)$. Aus Gl. (41) erhält man für die Verdünnungsentropie

$$
\Delta s_{2}=-R\left[\frac{\partial \lambda}{\partial n_{2}} \ln (1-\gamma)+\frac{1}{2 f(z)}\left(\Phi+T \frac{\partial \Phi}{\partial T}\right) \frac{n_{1}{ }^{2}}{n_{2}{ }^{2}}+\frac{p \Delta V_{2}}{R T}\right]
$$

und für die Verdünnungswärme

$\Delta H_{2}=-R T^{2}\left[\frac{1}{2} \frac{\partial}{f(z)} \frac{\partial \Phi}{\partial T} \frac{n_{1}{ }^{2}}{n_{2}{ }^{2}}+\frac{p}{R} \frac{\partial\left(\Delta V_{2} / T\right)}{\partial T}\right]$

Der osmotische Druck wird nach Gl. (41)

$$
\begin{aligned}
P=-\frac{R T}{\bar{V}_{2}}\left[\frac{\partial \lambda}{\partial n_{2}}\right. & \ln (1-\vartheta) \\
& \left.+\frac{\Phi}{2 f(z)} \frac{n_{1}{ }^{2}}{n_{2}{ }^{2}}+\frac{p \Delta V_{2}}{R T}\right] .
\end{aligned}
$$

Die früher eingeführte Funktion $\varphi$ ist nach dem Vorstehenden gegeben durch

$$
\varphi=\frac{\Phi}{2 f(z)} \frac{n_{1}^{2}}{n_{2}^{2}}+\frac{p \Delta V_{2}}{R T} .
$$

1V. Diskussion der statistischen Theorie der Solvatation

\section{a) Die Verdünnungswärme}

Die Erfahrung hat gezeigt, daß bei verdünnten Lösungen von FM (wie sie bei osmotischen Messungen zur Anwendung gelangen) $\Delta V_{2}=0$ ist. Man kann hier ferner den Molenbruch näherungsweise definieren durch $s_{1}=n_{1} / n_{2}$. Die Formel für die Verdünnungswärme lautet dann

$$
\lrcorner_{H_{2}}=-\frac{R T^{2}}{2 f(z)} \frac{\partial \Phi}{\partial T} N_{1}{ }^{2} .
$$

Daß die Verdünnungswärme in erster Näherung dem Quadrat des Molenbruches proportional ist, 
folgt bereits rein thermodynamisch aus der D u h e m - M a r g u l e s schen Gleichung. Der Vergleich mit den experimentellen Daten in Tab. 2 dient daher der Prüfung der Frage, wieweit diese Näherung brauchbar ist.

\begin{tabular}{|c|c|c|c|}
\hline $\begin{array}{c}c_{g} \\
\mathrm{~g} / l\end{array}$ & $N_{1} \cdot 10^{5}$ & $\begin{array}{c}\Delta H_{2} \exp \\
\cdot 10^{3} \\
\text { cal }\end{array}$ & $\begin{array}{c}\Delta H_{2 \text { theor }} \cdot 10^{3} \\
\text { cal }\end{array}$ \\
\hline \multicolumn{4}{|c|}{ Kautschuk-Toluol ${ }^{8}$} \\
\hline $\begin{array}{l}30,81 \\
41,78\end{array}$ & $\begin{array}{l}1,23 \\
1,67\end{array}$ & $\begin{array}{l}20 \\
45\end{array}$ & $\begin{array}{ll}20 & 23 \\
37 & 42\end{array}$ \\
\hline \multicolumn{4}{|c|}{ Kautschuk-Benzol ${ }^{20 *}$} \\
\hline $\begin{array}{r}4,4 \\
8,9 \\
17,9 \\
27,1 \\
36,6 \\
46,2 \\
66,1 \\
86,8 \\
120 \\
155\end{array}$ & $\begin{array}{l}.0,14 \\
0,28 \\
0,57 \\
0,86 \\
1,16 \\
1,47 \\
2,10 \\
2,76 \\
3,80 \\
4,92\end{array}$ & $\begin{array}{r}1,4 \\
5,5 \\
22,6 \\
54,6 \\
93,5 \\
148 \\
288 \\
500 \\
935 \\
1480\end{array}$ & $\begin{array}{rr}1,4 & 1,3 \\
5,7 & 5,1 \\
23,1 & 20,4 \\
53,0 & 47,4 \\
96,3 & 86,1 \\
154 & 137 \\
314 & 281 \\
542 & 485 \\
1031 & 922 \\
1726 & 1540\end{array}$ \\
\hline \multicolumn{4}{|c|}{ Nitrocellulose-Cyclohexanon ${ }^{\theta}$} \\
\hline $\begin{array}{l}16,27 \\
32,31 \\
61,14\end{array}$ & $\begin{array}{l}2,4 \\
4,8 \\
9,1\end{array}$ & $\begin{array}{r}-15 \\
-90 \\
-170\end{array}$ & $\begin{array}{rr}-15 & -14,4 \\
-59 & -56,9 \\
-212 & -204\end{array}$ \\
\hline \multicolumn{4}{|c|}{ Nitrocellulose-Aceton ${ }^{4,5}$} \\
\hline $\begin{array}{l}10 \\
20 \\
30\end{array}$ & $\begin{array}{l}0,90 \\
1,80 \\
2,70\end{array}$ & $\begin{array}{r}-4,5 \\
-20,6 \\
-39,3\end{array}$ & $\begin{array}{rr}-4,5 & -4,6 \\
-18,2 & -18,4 \\
-40,9 & -41,4\end{array}$ \\
\hline \multicolumn{4}{|c|}{ Polystyrol-Toluol 4,5 } \\
\hline $\begin{array}{l}10 \\
20 \\
30\end{array}$ & $\begin{array}{l}1,18 \\
\mathbf{2}, 36 \\
\mathbf{3 , 5 4}\end{array}$ & $\begin{array}{r}-4,7 \\
-16,6 \\
-34,4\end{array}$ & $\begin{array}{rr}-4,7 & -4,0 \\
-18,2 & -15,9 \\
-42,3 & -35,8\end{array}$ \\
\hline \multicolumn{4}{|c|}{ Acetylcellulose-Tetrachloräthan ${ }^{6}$} \\
\hline $\begin{array}{r}6,9 \\
14,8 \\
36,7 \\
72,9\end{array}$ & $\begin{array}{r}1,73 \\
3,68 \\
9,03 \\
18,29\end{array}$ & $\begin{array}{r}-6 \\
-15 \\
-155 \\
-440\end{array}$ & $\begin{array}{r}-6,0-4,3 \\
-27,2-19,3 \\
-163-116 \\
-671-476\end{array}$ \\
\hline \multicolumn{4}{|c|}{ Triacetylcellulose-Dioxan ${ }^{14}$} \\
\hline 2,36 & 0,26 & $-0,9$ & - \\
\hline 4,72 & 0,53 & $-1,2$ & - \\
\hline 6,22 & 0,70 & $+0,8$ & - \\
\hline 943 & 1,06 & $+9,8$ & - \\
\hline 13,88 & 1,55 & $+13,9$ & - \\
\hline 18,85 & 2,12 & $+25,2$ & 一 \\
\hline
\end{tabular}

* Die Daten wurden von den Autoren aus den Messungsergebnissen durch Interpolation gewonnen.

Tab. 2. Verdünnungswärmen pro Mol LM.

Die theoretischen $\Delta H_{2}$-Werte sind nach zwei Methoden berechnet: In der ersten Spalte ist der Proportionalitätsfaktor aus der niedrigsten Konzentration ermittelt; die zweite Spalte ist durch Ausgleichrechnung gewonnen. Die experimentellen Daten beruhen

${ }^{20}$ G. Ge e u. L. R. G. Tre lo a r, Trans. Faraday Soc. 38, 147 [1942]. auf osmotischen Messungen und haben daher eine verhältnismäßig hohe Fehlergrenze (etwa zwischen 10 und $50 \%$ ). Ge e u. Trelo a r ${ }^{20}$, welche die modernsten Messungen dieser Art ausgeführt haben, betonen ausdrücklich, daß die Meßgenauigkeit zu einer völlig gesicherten Ermittlung der Konzentrationsabhängigkeit von $\Delta_{H_{2}}$ nicht ausreicht. Mit diesem Vorbehalt ergeben sich folgende Schlüsse aus Tab. 2:

Bei den Systemen Kautschuk-Toluol, Nitrocellulose-Aceton, Polystyrol-Toluol werden die experimentellen Daten für alle aufgeführten Konzentrationen durch Gl. (43a) gut wiedergegeben. Bei Kautschuk-Benzol trifft dies bis zu etwa $N_{1}=2 \cdot 10^{-5} \mathrm{zu}$. Dagegen versagt hier die Formel bei höheren Konzentrationen, bei NitrocelluloseCyclohexanon, Acetylcellulose-Tetrachloräthan, Triacetylcellulose-Dioxan in dem ganzen Meßbereich. In diesen Fällen wird somit die Verdünnungswärme bereits wesentlich durch das kubische Glied und evtl. höhere Glieder bestimmt. Tab. 3 zeigt an dem Beispiel Acetylcellulose-Tetrachloräthan, daß sich hier die Messungsergebnisse durch eine Formel mit kubischem Glied schon recht gut darstellen lassen, und ferner, daß hier das kubische Glied bereits einen beträchtlichen Beitrag zur gesamten Verdünnungswärme liefert.

\begin{tabular}{|r|c|c|c|}
\hline$N_{1} \cdot 10^{5}$ & $\Delta H_{2 \text { exp }} \cdot 10^{3}$ & $\Delta H_{2 \text { ber }} \cdot 10^{8}$ & $B_{N_{1}{ }^{3}} \cdot 10^{3}$ \\
& cal & cal & cal \\
\hline & -6 & $-6,3$ & $+0,2$ \\
1,73 & -15 & $-27,3$ & $+2,4$ \\
3,68 & -155 & -144 & +35 \\
9,03 & -440 & -443 & +291 \\
18,29 & -25 \\
\hline
\end{tabular}

Tab. 3. Verdünnungswärme des Systems Acetylcellulose-Tetrachloräthan ${ }^{6}$ nach der Formel

$$
\Delta H_{2}=A_{N_{1}^{2}}+B N_{1}^{3} \text {. }
$$

Für die Diskussion des Faktors

$$
-\frac{R T^{2}}{2 f(z)} \frac{\partial \dot{\Phi}}{\partial T}
$$

und die Berechnung von $E$ ist es zweckmåßig, von vornherein konkrete Fälle ins Auge zu fassen und die Ergebnisse orientierender Rechnungen zu verwenden, da sich die Formeln für $\Phi$ dann wesentlich vereinfachen lassen. Es mögen zunächst die Systeme Kautschuk-Toluol und Kautschuk-Benzol betrachtet werden. In beiden Fällen ist $E<0$. Nach den Ergebnissen früherer Rechnungen ${ }^{2}$ (die, wie unten gezeigt wird, durch die Berücksichtigung der Solvatation nur unwesentlich beeinflußt werden) sind die FM hier sehr wenig beweglich, so daß man Gl. (36) anwenden kann. Ferner hat sich gezeigt, daß $n \varepsilon / k T$ so groß 
ist, daß die Exponentialfunktion nicht mehr durch eine rasch konvergierende Reihe dargestellt werden kann. Wegen $n \gg 1$ erhält man daher aus Gl. (36) und (43a) [da $f(z)=z$ ]

$$
\Delta_{H_{2}}=-\frac{z-2}{z} E \exp (-n E / R T) \frac{(n-1) \exp (-2 E / R T)-[n+\exp (-E / R T)]}{[\exp (-E / R T)-1]^{2}} N_{1}{ }^{2} \text { (Kautschuk). }
$$

Die Formel zeigt, daß hier die Verdünnungswärme nicht dem Quadrat des Volumenbruches proportional ist, sondern stärker mit der Kettenlänge wächst. Die erstere Annahme machen Gee u. Treloar ${ }^{20}$ im Anschluß an die für niedrigmolekulare Substanzen entwickelte Formel von Hildebrand ${ }^{21}$ u. Scatchard ${ }^{22}$; sie läßt sich aber aus ihren experimentellen Daten nicht verifizieren. Auch Kratky und M usil ${ }^{23}$ führen diesen Ansatz als erste Näherung ein.

Für das System Polystyrol-Toluol ist $E>0$. Auch hier kann die e-Funktion nicht entwickelt werden. Nach den früher ${ }^{3}$ mitgeteilten Rechnungen, die in Übereinstimmung sind mit den Untersuchungen von de B oer ${ }^{24}$, ist hier die Beweglichkeit ebenfalls sehr gering, so daß man wieder von Gl. (36) ausgehen kann. Man erhält dann für die Verdünnungswärme

$$
\Delta H_{2}=-\frac{1}{2} \frac{z-2}{z} n^{2} E \exp (-E / R T) N_{1}{ }^{2}
$$$$
\text { (Polystyrol-Toluol). }
$$

Hier ist somit die Verdünnungswärme in der Tat dem Volumenbruch proportional. Experimentelles Material zur Prüfung dieser Aussage liegt noch nicht vor.

Bei Nitrocellulose-Aceton ist ebenfalls $E>0$. Uber die Beweglichkeit gehen hier die Ansichten noch weit auseinander. Während sie nach $\mathrm{H}$ e r m a n s ${ }^{25,26} \mathrm{sehr}$ groß ist, nehmen $\mathrm{D}$ oty u. $\mathrm{M} \mathrm{ark}{ }^{27}$ an, daß hier das FM einem elastischen Draht zu vergleichen sei. Für Methylcellulose nehmen auch Sig n e r und $\mathrm{T}$ a v e ${ }^{28}$ auf Grund ihrer Messungen mit der Ultrazentrifuge an, daß starke innere Beweglichkeit nicht vorhanden ist. Die osmotischen Messungen an NitrocelluloseAceton ${ }^{10}$ deuten aber auf eine gewisse Beweglichkeit hin $^{3}$. Die nähere Untersuchung zeigt in Übereinstimmung damit, daß Gl. (43c) nicht anwendbar ist. Für

${ }^{21} \mathrm{H}$ ild e brand, Solubility of Non-Electrolytes, New York [1936].

${ }_{22}$ Scatchard, J. Amer. Chem. Soc. 56, 995 [1934].

${ }^{23} \mathrm{Kratky}$ u. Musil, Z. Elektrochemie 43, 326 [1937].

${ }_{24}$ d e B o er, Trans. Faraday Soc. 32, 10 [1936].

${ }_{25}$ Hermans, Kolloid. Z. 102, 169 [1942].

${ }^{26} \mathrm{Hermans}$, Contribution to the Physics of Cellulose Fibres, Amsterdam 1946. geringe Beweglichkeit erhält man mit Gl. (38)

$$
\Delta H_{2}=-\frac{1}{2} \frac{z-2}{z}(z-1)^{\frac{n}{x}-1} n^{2} E \exp (-E / R T) N_{1}{ }^{2}
$$

In Tab. 4 sind die jeweils nach den angegebenen Formeln berechneten $E$-Werte zusammen mit den benutzten $z$-Werten aufgeführt ${ }^{29}$.

\begin{tabular}{|c|c|cc|c|}
\hline System & $n$ & \multicolumn{1}{c|}{$\begin{array}{c}\boldsymbol{z} \\
\text { (II. Näher.) }\end{array}$} & $\begin{array}{c}E \\
\text { cal/Mol }\end{array}$ \\
\hline Kautschuk-Toluol & 3967 & 2,35 & $(2,26)$ & 0,842 \\
Kautschuk-Benzol & 4114 & $\mathbf{2 , 3 7}$ & $(2,35)$ & 1,006 \\
Polystyrol-Toluol & $\mathbf{8 6 0}$ & $\mathbf{3 , 6}$ & $\mathbf{5 9 5}$ \\
\hline
\end{tabular}

Tab. 4. Molare Wechselwirkungsenergien pro Baustein der FM.

Die $z$-Werte für die Kautschuklösungen sind aus der Verdünnungsentropie berechnet ${ }^{2}$; der für Polystyrol-Toluol benutzte ist der niedrigste nach Gl. (43c) mögliche, er liegt über dem aus den osmotischen Messungen berechneten $(z=2,28)^{3}$.

Die zunächst auffallende Tatsache, daß $E$-Werte, die sich um zwei Größenordnungen unterscheiden, auf Verdünnungswärmen von der gleichen Größenordnung führen, erklärt sich nach der Rechnung dadurch, daß im Falle $\Delta_{H_{2}}<0$ im wesentlichen die Konfigurationen mit $m=1$, im Falle $\Delta H_{2}>0$ die mit $m>1$ zur Verdünnungswärme beitragen. Der hohe $E$-Wert von Polystyrol-Toluol erscheint für eine Mischung ähnlicher Kohlenwasserstoffe wenig wahrscheinlich (vergl. dazu auch ${ }^{2}$ ).

\section{b) Die Verdünnungsentropie}

Die Formel für die Verdünnungsentropie kann für die oben diskutierten Fälle geschrieben werden:

27 Doty u. Mark, Ind. Eng. Chem. 38, 682 [1946]. $28 \mathrm{~S}$ i g n er u. Tave 1, Helv. chim. Acta 21, 535 [1938].

${ }_{29}$ Die Auswertung von Gl. (43d) setzt Kenntnis der Größe $x$ voraus, die aus Messungen von $\Delta_{\mathbf{2}}$ an polymerhomologen Reihen zu entnehmen wäre. Solche Messungen liegen bisher noch nicht vor. 


$$
\begin{aligned}
& \Delta s_{2}=R s_{1}\left\{1+\frac{z-2}{z} n^{2}\left[\frac{1}{2}-\frac{1}{n^{2}} \exp (-n E / R T)\right.\right. \\
& \left.\left.\frac{[\exp (-2 E / R T)-1][1+n E / R T]-E / R T[\exp (-2 E / R T)+\exp (-E / R T)]}{[\exp (-E / R T)-1]^{2}}\right] N_{1}\right\} \\
& \text { (Kautschuk) } \\
& \Delta s_{2}=R N_{1}\left\{1+\frac{\dot{z}-2}{z} n^{2}\left[1-\frac{1}{2} \exp (-E / R T)(1+E / R T)\right] N_{1}\right\} \quad \text { (Polystyrol-Toluol) } \\
& \Delta s_{2}=R N_{1}\left\{1+\frac{z-2}{z} n^{2}\left[\frac{x z}{2 n}\left[1-\left(\frac{z-1}{z}\right)^{\frac{n}{x}}\right]+\frac{1}{2}(z-1)^{\frac{n}{x}-1}[1-\exp (-E / R T)(1+E / R T)]\right] N_{1}\right\}
\end{aligned}
$$

Aus Gl. (42b) und (42c) ergibt sich die bemerkenswerte Folgerung, daß der Solvatationsanteil der Verdünnungsentropie stets positiv ist*. Die unter II. erwähnte „Kompensation“ der athermischen Verdünnungsentropie durch die Solvatation ist also nur dann möglich, wenn schon das kubische Glied der Verdünnungswärme ins Gewicht fällt, wie es bei dem System AcetylcelluloseTetrachloräthan der Fall ist (Tab.3). Tab. 5 ent- hält für einige Systeme den Solvatationsanteil der Verdünnungsentropie $\Delta s_{2 \text { solv }}$, die daraus und aus den experimentellen Werten $\Delta s_{2 \exp }$ berechnete

\begin{tabular}{|c|c|c|c|c|c|c|}
\hline $\begin{array}{c}c_{g} \\
\mathbf{g} / l\end{array}$ & $N_{1} \cdot 10^{5}$ & $\begin{array}{c}\Delta s_{2 \text { exp }} \cdot 10^{5} \\
\text { cal } / \text { grad }\end{array}$ & $\begin{array}{c}\Delta s_{2} \text { solv } \cdot 10^{5} \\
\text { cal } / \text { grad }\end{array}$ & $\begin{array}{c}\Delta 8_{2 \text { ath }} \cdot 10^{5} \\
\mathrm{cal} / \mathrm{grad}\end{array}$ & $\begin{array}{c}\Delta s_{2 \text { id }} \cdot 10^{5} \\
\mathrm{cal} / \mathrm{grad}\end{array}$ & $\begin{array}{c}\Delta s_{2 \text { ath theor }} \cdot 10^{5} \\
\text { cal/grad }\end{array}$ \\
\hline \multicolumn{7}{|c|}{ Kautschuk-Toluol ${ }^{8}$} \\
\hline $\begin{array}{l}30,81 \\
41,78\end{array}$ & $\begin{array}{l}1,23 \\
1,67\end{array}$ & $\begin{array}{l}36 \\
63\end{array}$ & $\begin{array}{r}5,8 \\
10,8\end{array}$ & $\begin{array}{l}30,2 \\
52,2\end{array}$ & $\begin{array}{l}2,4 \\
3,3\end{array}$ & $\begin{array}{l}29,4 \\
53,0\end{array}$ \\
\hline \multicolumn{7}{|c|}{ Kautschuk-Benzol ${ }^{20}$} \\
\hline $\begin{array}{r}4,4 \\
8,9 \\
17,9 \\
27,1 \\
36,6 \\
46,2\end{array}$ & $\begin{array}{l}0,14 \\
0,28 \\
0,57 \\
0,86 \\
1,16 \\
1,47\end{array}$ & $\begin{array}{c}0,86 \\
3,4 \\
14 \\
34 \\
63 \\
105\end{array}$ & $\begin{array}{c}0,39 \\
1,6 \\
6,4 \\
15 \\
27 \\
43\end{array}$ & $\begin{array}{c}0,47 \\
1,8 \\
7,6 \\
19 \\
36 \\
62\end{array}$ & $\begin{array}{l}0,28 \\
0,56 \\
1,13 \\
1,71 \\
2,3 \\
2,9\end{array}$ & $\begin{array}{l}0,78 \\
2,6 \\
9,3 \\
20 \\
36 \\
57\end{array}$ \\
\hline \multicolumn{7}{|c|}{ Polystyrol-Toluol ${ }^{4,5}$} \\
\hline $\begin{array}{l}10 \\
20 \\
30\end{array}$ & $\begin{array}{l}1,18 \\
2,36 \\
3,54\end{array}$ & $\begin{array}{l}2,55 \\
5,0 \\
7,3\end{array}$ & $\begin{array}{r}1,2 \\
4,8 \\
10,8\end{array}$ & $\begin{array}{l}1,35 \\
0,2 \\
-3,5\end{array}$ & $\begin{array}{l}2,4 \\
4,7 \\
7,0\end{array}$ & \\
\hline
\end{tabular}
athermische Verdünnungsentropie $\Delta s_{2}$ ath und schließlich die aus der Theorie der athermischen Lösung berechneten Werte $\Delta s_{2 \text { ath theor. Außerdem }}$ sind noch die für die ideale Lösung berechneten Werte $\Delta s_{2 \text { id }}$ aufgeführt.

Tab.5. Verdünnungsentropien pro Mol LM.

Die Tabelle zeigt, daß die Solvatation bei Kautschuk-Toluol nur wenig, bei Kautschuk-Benzol dagegen schon beträchtlich (ca. 50\%) zur Verdünnungsentropie beiträgt. Die Zahlen der letzten Spalte stimmen im allgemeinen gut mit den für $\Delta s_{2 \text { ath }}$ ermittelten Werten überein.

Nur bei den kleinsten Konzentrationen von Kautschuk-Benzol zeigen sich Abweichungen. Dies wurde bereits von Gee und Treloar 20 bemerkt; da aber hier schon die Fehlergrenze der osmotischen Messung $20 \%$ beträgt, muß die Realität des Effektes vorläufig bezweifelt werden. Die aus der letzten Spalte

* Anm. b. d. Korrektur: Zu dem gleichen Ergebnis führen die Theorien von Or sowie Alfrey u. Doty (s. Anm. S. 287). berechneten $z$-Werte weichen etwas ab von den zur Berechnung von $E$ und $\Delta s_{2}$ solv benutzten; sie finden sich ebenfalls (eingeklammert) in Tab. 4. Man könnte mit den neuen Werten in sukzessiver Näherung die Rechnung wiederholen, doch dürfte sich dies nur bei wesentlicher Erhöhung der Meßgenauigkeit lohnen.

Die Theorie der athermischen Lösung läßt sich so quantitativ exakt prüfen. Die Anwendung der Theorie auf die Messungen an Polystyrol-Toluol und Nitrocellulose-Aceton führt zu unmöglichen Ergebnissen. Dieser Widerspruch bedarf noch der Aufklärung durch neue Experimente.

Während bei der athermischen Lösung starrer FM die $\Delta s_{2} / c_{g}$-Kurven polymerhomologer Reihen 
parallel verlaufen, trifft dies für den Solvatationsanteil nur im Falle $\Delta_{2}<0 \mathrm{zu}[\mathrm{Gl} .(42 \mathrm{~b})]$. Dagegen nimmt die Steigerung mit dem Polymerisationsgrad $\mathrm{zu}$, wenn $\boldsymbol{\Delta H}_{2}>0$ ist. In den Ergebnissen von Gee und Trelo a r ${ }^{20}$ findet sich dieser Effekt angedeutet; er kann aber noch nicht als gesichert gelten, da die Annahme über $\Delta_{H 2}$, wie erwähnt, mit Gl. (43 b) in Widerspruch steht.

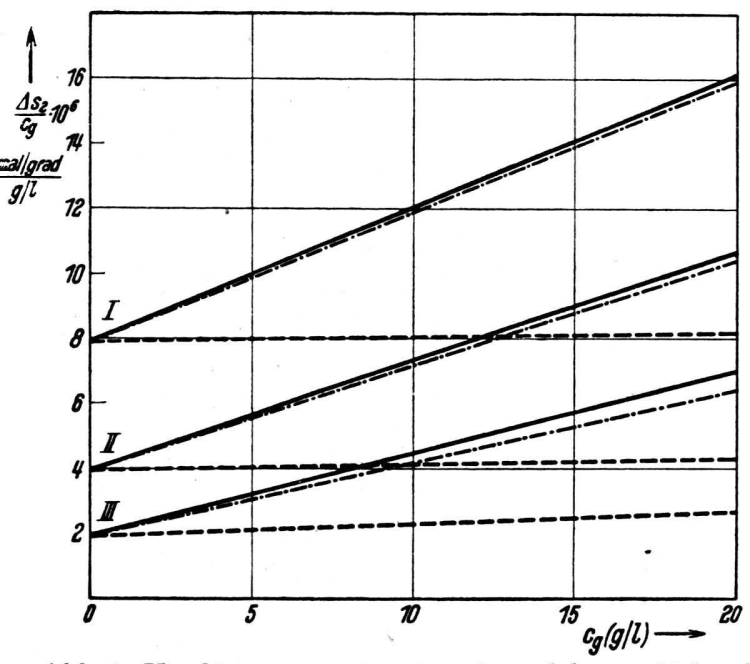

Abb. 1. Verdünnungsentropie schwach beweglicher FM [Gl. $(43 \mathrm{c})]$.

$$
\begin{array}{ll}
\frac{\left(\Delta s_{2 \text { ath }}+\Delta s_{2 \text { solv }}\right)}{c_{g}} & -\cdots--1 \Delta \\
\ldots \frac{\left(\Delta s_{2} \text { id }+\Delta s_{2} \text { solv }\right)}{c_{g}} & \Delta H_{2}<0 . \\
\text { I: } n=250 . \text { II }: n=500 . & \text { III }: n=1000 .
\end{array}
$$

Bei beweglichen FM nimmt die Steigung von $\Delta s_{2}$ ath $/ c$ mit dem Polymerisationsgrad $\mathrm{ab}^{2,3}$, die von $\Delta s_{\text {, solv }} / c_{g}$ dagegen $\mathrm{zu}$; das Ergebnis hängt daher von dem Verhältnis der beiden Anteile ab. In Abb. 1 sind beide Anteile und die resultierenden Kurven für ein konstruiertes Beispiel nach Gl. (42c) dargestellt.

\section{c) Der osmotische Druck}

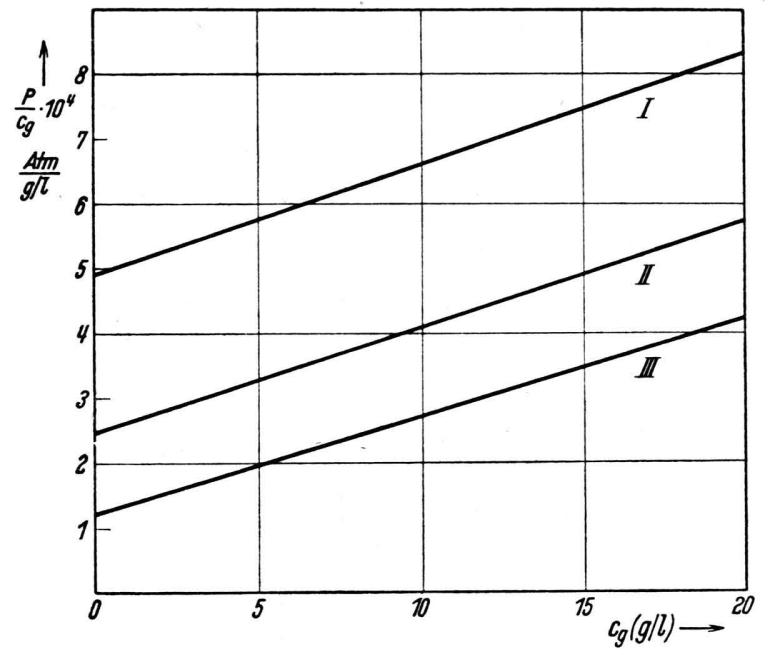

Abb. 2. Osmotischer Druck starrer FM [Gl. (44a)]. $\Delta H_{2}<0$.

I: $n=500 . \quad$ II $: n=1000 . \quad$ III $: n=2000$.

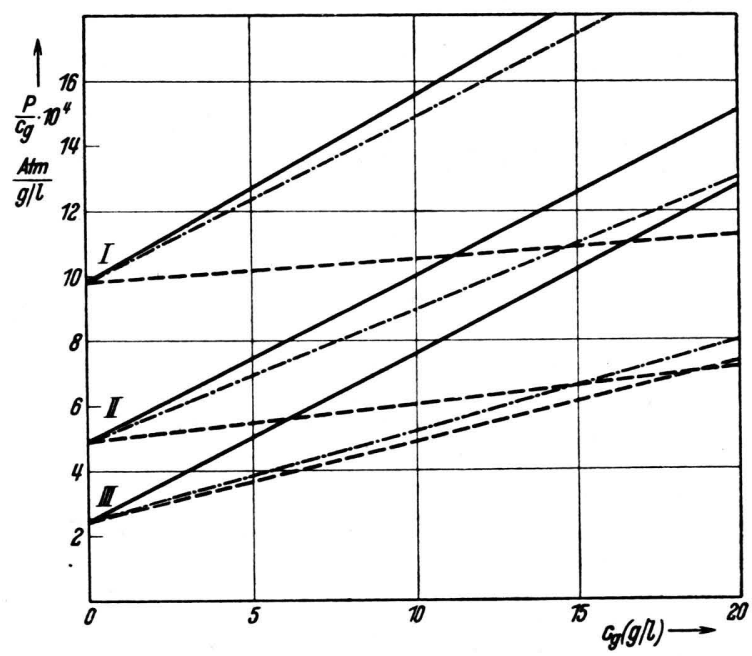

Abb. 3. Osmotischer Druck schwach beweglicher FM [Gl. (44 c)]. $\Delta \mathrm{H}_{2}<0$.

$$
\left(P_{\text {ath }}+P_{\text {solv }}\right) / c_{g} \quad-\cdot-\cdot P_{\text {ath }} / c_{g}
$$$$
\text { -...... }\left(P_{\text {id }}+P_{\text {solv }}\right) / c_{g}
$$

I: $n=250$. II: $n=500$. III $: n=1000$.

Die Formel für den osmotischen Druck lautet für die oben diskutierten Fälle

$$
P=\frac{R T}{\bar{V}_{2}} N_{1}\left\{1+\frac{z-2}{z} n^{2}\left[\frac{1}{2}-\frac{1}{n^{2}} \exp (-n E / R T) \frac{\exp (-E / R T)+1}{\exp (-E / R T)-1}\right] N_{1}\right\} \quad \text { (Kautschuk) }
$$

$$
P=\frac{R T}{\bar{V}_{2}} N_{1}\left\{1+\frac{z-2}{z} n^{2}\left[1-\frac{1}{2} \exp (-E / R T)\right] N_{1}\right\} \quad \text { (Polystyrol-Toluol) }
$$

$P=\frac{R T}{\bar{V}_{2}} N_{1}\left\{1+\frac{z-2}{z} n^{2}\left[\frac{x z}{2 n}\left[1-\left(\frac{z-1}{z}\right)^{\frac{n}{x}}\right]+\frac{1}{2}(z-1)^{\frac{n}{x}-1}[1-\exp (-E / R T)]\right] N_{1}\right\}$ 
Aus diesen Gleichungen folgt zunächst, in Übereinstimmung mit der thermodynamischen Theorie (II) und der Erfahrung (Tab.1), daß die Abweichungen von der idealen Lösung im Falle $\Delta_{H_{2}}>0$ für die Verdünnungsentropie größer sind als für den osmotischen Druck, während für $\Delta H_{2}<0$ das Gegenteil zutrifft. Aus Gl. (44a) folgt weiter, daß hier die Steigung der $P / c_{g}-\left(c_{g}\right)$ Kurven polymerhomologer Reihen mit dem Polymerisationsgrad abnimmt. Für $\Delta_{2}<0$ verlaufen dagegen die Kurven starrer FM parallel [Gl. (44b)], während bei beweglichen FM, soweit die Solvatation in Betracht kommt, die Steigung mit dem Polymerisationsgrad zunimmt [Gl. (44e)]. Damit ist nachgewiesen, daß die schon früher ${ }^{3}$ diskutierte Abnahme der Steigung mit dem Polymerisationsgrad im Falle $\Delta H_{2}<0^{30}$ tatsächlich die Theorie der athermischen Lösung bestätigt und nicht durch die Solvatation erklärt werden kann. In Abb. 2 und 3 sind die Verhältnisse für zwei Beispiele nach Gl. (44a) und (44c) dargestellt.

Auf Grund der obigen Beziehungen ist es mit Hilfe der bisher gewonnenen Daten möglich, den osmotischen Druck vollständig vorauszuberechnen und damit die Theorie quantitativ zu prüfen. Tab. 6 enthält die experimentellen und berechneten Werte.

Bei den Kautschuklösungen wird die Theorie gut bestätigt. Dagegen ergibt die Anwendung auf Polystyrol-Toluol auch hier ein unmögliches Resultat.

Der eigentliche Kern der Schwierigkeit liegt darin, daß $\Delta_{H_{2}}$ hier dem quadratischen Gesetz gehorcht. Ganz ähnlich liegt der Fall auch bei dem System Nitrocellulose-Aceton. Die von G. V. S ch u l z ${ }^{4,5}$ zur Berechnung des osmotischen Druckes verwendete Formel

$$
P=\frac{R T}{M_{1}} c_{g}-\frac{\Delta H_{2}}{\bar{V}_{2}}
$$

${ }^{30}$ Dies trifft von den in ${ }^{3}$ diskutierten Beispielen jedenfalls für Polystyrol-Toluol und NitrocelluloseAceton zu.

\begin{tabular}{|c|c|c|c|c|}
\hline $\begin{array}{c}c_{g} \\
\mathrm{~g} / l\end{array}$ & $N_{1} \cdot 10^{5}$ & $\begin{array}{l}P_{\text {exp }} \\
\cdot 10^{3} \\
\text { Atm }\end{array}$ & $\begin{array}{l}P_{\text {theor }} \\
\cdot 10^{3} \\
\text { Atm }\end{array}$ & $\begin{array}{c}\left(P_{\text {solv }}\right. \\
\left.+P_{\text {id }}\right) 10^{3} \\
\quad \text { Atm }\end{array}$ \\
\hline \multicolumn{5}{|c|}{ Kautschuk-Toluol ${ }^{8}$} \\
\hline $\begin{array}{l}30,81 \\
41,78\end{array}$ & $\begin{array}{l}1,23 \\
1,67\end{array}$ & $\begin{array}{l}29,8 \\
50,9\end{array}$ & $\begin{array}{l}32,7 \\
58,9\end{array}$ & $\begin{array}{l}1,6 \\
1,4\end{array}$ \\
\hline \multicolumn{5}{|c|}{ Kautschuk-Benzol ${ }^{20}$} \\
\hline $\begin{array}{l}0,49 \\
0,98 \\
1,70 \\
2,36\end{array}$ & $\begin{array}{l}0,157 \\
0,310 \\
0,540 \\
0,744\end{array}$ & $\begin{array}{c}0,68 \\
2,5 \\
7,8 \\
16,6\end{array}$ & $\begin{array}{r}1,2 \\
3,7 \\
10,2 \\
18,5\end{array}$ & $\begin{array}{r}0,32 \\
0,41 \\
0,13 \\
-0,53\end{array}$ \\
\hline \multicolumn{5}{|c|}{ Polystyrol-Toluol 4,5 } \\
\hline $\begin{array}{l}10 \\
20 \\
30\end{array}$ & $\begin{array}{l}1,18 \\
2,36 \\
\mathbf{3 , 5 4}\end{array}$ & $\begin{array}{l}4,75 \\
12,5 \\
22\end{array}$ & $\begin{array}{l}10,7 \\
38,6 \\
83,6\end{array}$ & $\begin{array}{r}5,5 \\
17,6 \\
36,3\end{array}$ \\
\hline
\end{tabular}

Tab. 6. Osmotische Drucke.

gilt, wie man durch Vergleich mit Gl. (44b) leicht feststellt, nur unter den beiden Voraussetzungen, daß $\Delta s_{2}$ ath $=\Delta s_{2 \text { id }}$ und $E / R T \ll 1$. Nach den hier und früher durchgeführten Untersuchungen treffen beide Voraussetzungen nicht $\mathrm{zu}$.

Die letzte Spalte der Tab. 6 enthält Werte für den osmotischen Druck, die aus dem van 't $\mathrm{H}$ of $\mathrm{f}$ schen Gesetz unter Hinzunahme der Solvatation berechnet sind. Sie zeigt, daß bei den Kautschuklösungen die Solvatation überhaupt nicht zur Erklärung der experimentellen Werte dienen kann, weil sie einen negativen Anteil ergibt. Bei Polystyrol-Toluol dagegen liefert sie schon für sich zu hohe Werte.

Formal beruht die Abweichung hier darauf, daß die Verdünnungswärme einen zu hohen $z$-Wert erzwingt. Wie früher ${ }^{3}$ gezeigt, kann der osmotische Druck von Polystyrol-Toluol nach der Theorie der athermischen Lösung mit $z=2,28$ gut dargestellt werden.

Bei den Kautschuklösungen wird somit der früher ${ }^{2}$ gezogene Schluß bestätigt, daß ihre thermodynamischen Eigenschaften in erster Linie durch Größe und Gestalt der gelösten FM bestimmt werden. Auch die Auffassung, daß hier die FM nur wenig beweglich sind, ist mit den vorstehenden Untersuchungen in guter Übereinstimmung.

Der Gesellschaft der Freunde der Universität Heidelberg danke ich für die Gewährung eines Forschungsstipendiums. 